\title{
ARTS ET INDUSTRIES DE LA CROYANCE : QUAND LE LANGAGE FAIT SON CINÉMA...
}

\author{
Frédéric Lambert ${ }^{1}$
}

Une proposition, sous forme de programme pour interroger le mot " croyance » souvent trop épais pour ses usagers. Des exemples, sous forme de courtes fables, pour comprendre que la croyance n'est pas une soumission à une religion mais une intelligence face aux langages des médias-cultures. La croyance, interrogée dans les cadres pluriels des sciences humaines et sociales, ce serait cette faculté de nous jouer des langages et poursuivre avec notre société les récits qu'il nous faut inventer chaque jour pour vivre ensemble.

Ce texte propose une définition de la « croyance» pour comprendre comment la sémiologie, informée par la psychanalyse, l'anthropologie et l'histoire, peut se permettre d'entendre ce mot, souvent trop grand pour ses usagers.

Avec Paul Veyne, Bruno Latour, Octave Mannoni et Michel de Certeau, nous décrivons le programme que nous nous imposons pour interroger la croyance considérée comme un temps d'intelligences partagées. En effet, le mot croyance est souvent pris dans le paradigme de la manipulation, de l'aveuglement, de l'adhésion d'un groupe. Le mythe, l'énoncé idéologique, les discours pris dans l'ordre des institutions qui

1 Frédéric Lambert est Responsable du Master Médias, langages et sociétés à 1'Institut Français de Presse, Université Paris 2, et directeur du Centre d'analyses et de recherches interdisciplinaires sur les médias (CARISM), Sorbonne Universités.

Recherches en communication, $\mathrm{n}^{\circ} 38$ (2012). 
les communiquent, diffuseraient leurs poisons au détriment de la liberté de l'individu et des peuples. C'est là une proposition pour nous erronée qui considère la croyance comme un contenu et non comme un geste partagé. C'est pour cette raison que l'une des propositions de Paul Veyne pour penser la croyance nous convient : « Il faut refuser la croyance de l'autre comme acquise, mais la considérer comme un petit arrangement entre lui et sa société » (Veyne, 1969). Ainsi la croyance ne serait pas définitive : même si les mythes, les énoncés idéologiques, les discours de l'ordre se figent un moment dans les langages d'une propagande terriblement efficace, même si ces mêmes langages prétendent, grâce à la rhétorique de l'Éternité être définitifs, la croyance reste un « petit arrangement provisoire » entre un individu et la société dans laquelle il vit.

Pour illustrer cette proposition, ce texte débute par un cas concret, le film Avatar et son succès mondialisé. Objet des industries culturelles, film de fiction, il écrit dans les langages du divertissement un grand récit écologique (une planète où les habitants vénèrent leur Nature) et un grand récit contre le colonialisme industriel (les terriens viennent tout détruire et tuent pour implanter leurs usines d'extraction d'un minerai précieux). Mais très rapidement, on voit l'information se saisir de ce film pour parler des actualités bien réelles celles-ci : la déforestation au Brésil, le mur de séparation entre Israël et la Palestine, des pollutions en Inde. On observe alors comment nos récits circulent entre l'information et la communication d'une part, le fictionnel et le factuel d'autre part. Et c'est précisément dans ces échanges que s'exercent nos croyances.

Pour appuyer notre hypothèse, nous nous référons ensuite aux quatre auteurs cités plus haut qui nous semblent avoir fondé une «théorie de la croyance »: Paul Veyne, Bruno Latour, Octave Mannoni, Michel de Certeau. Chacun, à la place qu'il occupe dans le champ des sciences sociales, relativise le paradigme de l'aveuglement pour penser la croyance comme une forme de déni : " je sais bien, mais quand même ». Le déni, c'est cette capacité humaine à négocier avec la proposition qui lui est faite : «je sais bien que mes récits sont fabriqués dans les langues provisoires de mon groupe, mais quand même je vais accepter leurs propositions pour vivre en société ».

Enfin, autour de la question du statut du spectateur au cinéma, nous proposons de considérer la croyance comme une capacité à naviguer entre le fictionnel et le factuel, entre les discours de l'information et 
ceux de la communication, entre les différentes sphères de production culturelles : ainsi se ramifient les médiacultures. Arts, industries culturelles, médias, réseaux tissent leurs références dans un même « fond commun », celui de la communauté et ses nécessaires fabriques de lieux communs. Si les lieux communs favorisent les situations de croyance, nos exercices quotidiens de spectateur (passage d'un support à un autre, d'une situation de communication à une autre, d'un lieu de discours à un autre) finissent par former une intelligence face aux propositions qui nous sont faites. La pluralité des formes de langages relativise les propositions que ces derniers nous font. C'est en ce sens que la culture et les médias sont des lieux de résistance face aux propagandes : en nous exerçant et en multipliant les situations de déni, les médiacultures favorisent paradoxalement notre esprit critique.

Conscient de la position pour le moins optimiste de cette thèse, la conclusion de ce texte interroge ce qu'elle signifie en termes moral et politique.

\section{Nos cinémas, avatars d'Avatar}

Avatar. En 3D, c'est presque de la réalité augmentée, et c'est une science fiction d'un nouveau genre, intitulé planet opera, biodégradable, avec des hommes en bleu ${ }^{1}$.

Le fait que je m'identifie au héros avec ses grandes oreilles et sa peau bleue, que je me laisse émouvoir par une scène de réincarnation, que j'oublie l'ensemble du dispositif de fabrication et la dimension fictionnelle du film participe au plaisir d'aller au cinéma. Je sais les effets spéciaux, je sais les langages, je sais le scénario et ses grosses ficelles produites dans un savoir-faire hollywoodien, rien n'y fait. Au cinéma, nous sommes libres d'entrer en croyance, cet état de vacances, cette intelligence qui nous laisse adhérer au récit qui nous est conté et participer collectivement à l'émotion garantie par quatre cent soixante

1 Ce film est l'un des plus coûteux de toute l'histoire du cinéma. Selon The New York Times (Michael Cieply, «A Movie's Budget Pops From the Screen », 8 novembre 2009), le budget d'Avatar s'élèverait à 460 millions de dollars (dont 145 en budget publicitaire). Mais son succès public lui a permis de dégager des bénéfices après seulement dix jours d'exploitation et de récolter plus de 2,7 milliards de dollars de recettes. 
millions de dollars investis et deux milliards sept cent mille dollars de recette. Oui, à ce prix, nous pouvons exiger quelques plans planants.

Mais nos croyances ne s'arrêtent pas une fois nos lunettes 3D enlevées. Et c'est là le propre de nos sociétés de faits et de fictions. Trois exemples liés à ce film me permettent de dire comment la croyance dans ce dernier et, plus largement dans les productions filmiques, s'invite aujourd'hui dans tous les domaines où l'image tient une bonne place : art, industries culturelles, médias. Ici, ce n'est plus le réel qui nourrit la fiction, c'est la fiction qui nourrit nos réels. Et pour être en société, il faut nous adapter. Nos langages et les récits qu'ils racontent sont tissés dans les registres pluriels des médiacultures ${ }^{1}$ qui occupent tout le champ de la production des images. Ce n'est plus seulement « Pourquoi la fiction? » (Schaeffer, 1999) qu'il nous faut poser comme question, mais « Pourquoi le réel traversé de fictions »?

Ainsi en février 2010, la légende de la photographie de jeunes palestiniens et de militants israéliens du mouvement La paix maintenant, peints en bleu permet de lire : « [...] Déguisés en Na’vi, les créatures du film Avatar de James Cameron, des activistes célèbrent le début des travaux modifiant le tracé de la clôture de sécurité israélienne à Bilin, près de Ramallah, en Cisjordanie » (Anonyme 1, 2010, p. 6-7).

Ainsi, en février 2010, sous la photographie de représentants de la tribu des Dongria en Inde tenant une banderole sur laquelle il est écrit «We are Dongria Kondh. Vedanta can not take our moutain ${ }^{2} »$, on peut lire : « La tribu des Dongria Kondh vit le scénario du film Avatar. Le peuple autochtone qu'un géant minier veut expulser de sa terre sacrée a lancé un appel à James Cameron. La tribu des Dongria Kondh manifeste

1 J'emprunte ce terme aux travaux d'Éric Maigret et d'Éric Macé (Macé, 2006, p. 2935). Dans la filiation des Culural Studies, ce concept régénère la relation entre médias et culture et, plus largement, entre cultures d'élites et cultures populaires. L'observation des langues médiatiques culturelles et artistiques, leurs échanges, leurs emprunts, nous placent sans réserve dans cette perspective. La complexité des objets médiaculturels interroge profondément une intelligence de la réception qui nécessairement construit une vigilance face aux propositions plurielles des nouveaux langages et des nouveaux contextes de nos images et de nos imaginaires médiatiques.

2 « Nous sommes les Dongria Kondh. L'entreprise Vedanta ne doit pas nous prendre nos montagnes » 
contre l'installation de la mine du groupe Vedanta Ressources », géant minier britannique exploitant les gisements de bauxite polluant les cours d'eau et menaçant la santé des habitants (Anonyme 2, 2010, p. 6).

Ou encore, sous le titre " [...] Un millions d'arbres pour Avatar», le journal Le Monde raconte comment James Cameron soutient la lutte des indiens Xingu en Amazonie, menacés par la déforestation.

La fiction, celle d'une industrie culturelle lourde, vient donc nous raconter nos réels. Ces derniers n'y suffisent plus pour nous informer, il nous faut des communications scénarisées et mondialisées. Ainsi nous devenons trois fois croyants : spectateurs du film, nous sommes dans l'aventure, aimons, vivons, mourons, avec les personnages. Pris dans les discours d'accompagnements (toute la communication autour du film, pour un budget d'environ 200 millions de dollars et ses relais médiatiques) et ceux de la sphère publique (émissions, expositions, articles et discours autour du film dans l'espace public), nous adhérons au grand récit du film, sa superproduction idéologique verte et bleue, à sa Terre menacée, au grand récit écologique de ce planet opera, et traduisons la fiction interplanétaire à l'échelle de notre écologie terrestre. Citoyens enfin, nous entendons la métaphore politique de résistance que le film nous dit et qui se répand comme une narration obligée pour défendre tous les territoires occupés et menacés, les libertés bafouées en Inde, en Palestine, au Brésil. Qu'il nous faille entrer en fiction pour " ré-enchanter » le réel, nous le savons depuis l'enfance. Mais aujourd'hui, pour réactiver le réel, le réactualiser, nous nous référons aux fictions. Le peuple palestinien, les Dongria Kondh, les Xingu d'Amazonie ne pourraient-ils se penser que dans le filtre de nos divertissements? Nos cinémas, nous ne les arrêtons plus aux seules frontières de la fiction et de ses dispositifs de croyance circonscrits à la salle de cinéma. Nos cinémas se jouent en plein air, dans la rue, sur les pages de l'information, et nos réels médiactivés nous proposent une belle séance de projection du monde.

Voici mon hypothèse : la croyance aujourd'hui serait cette capacité à passer d'un espace discursif à un autre, d'une situation d'énonciation à une autre, d'un récit à un autre, où écritures fictionnelles et écritures factuelles nous séduisent de concert. Rien de grave. La croyance comme une intelligence : en voici le programme. 


\section{Demandez le programme : intelligences de la croyance}

Projet pluriel, la volonté d'inscrire la croyance dans le paradigme de l'intelligence nous est suggérée par les travaux de Paul Veyne en histoire, de Bruno Latour et Michel de Certeau en anthropologie et d'Octave Mannoni en psychanalyse.

Paul Veyne, dans Les grecs ont-ils cru à leurs mythes? établit un programme pour penser la croyance. Paul Veyne construit une théorie du doute que chaque chevalier de la liberté devrait méditer avant de partir en croisade : «Les hommes ne trouvent pas la vérité : ils la font, comme ils font leur histoire, et elles le leur rendent bien » (Veyne, 1983, p. 12). Les grecs ont-ils cru à leurs mythes ? Cette question demande une méthode. Et cette méthode prend chez Paul Veyne la forme d'un programme :

- Il faut refuser la croyance de l'autre comme acquise, mais la considérer comme un petit arrangement entre lui et sa société.

- Il faut associer le verbe « croire » au concept de « vérité », et la vérité est que la vérité varie.

- Il faut définir le mythe.

- Il faut observer les sources anonymes ou collectives du récit historique (ou du récit médiatique) qui cautionnent soit dans l'énoncé, soit dans l'énonciation la vérité supposée du mythe.

- Il faut regarder comment le croyant navigue entre les récits de la fiction et les récits de la réalité.

- Il faut comprendre enfin, face aux dogmes et aux obéissances que des classes sociales acceptent partiellement ou subissent totalement, à qui profite que l'on croie.

« Les Grecs, écrit Paul Veyne, croient et ne croient pas à leurs mythes ; ils y croient mais ils s'en servent et ils cessent d'y croire là où ils n'y ont plus d'intérêt ; (...) et tous les peuples donnent un coup de pouce à leurs oracles ou à leurs indices statistiques pour se faire confirmer ce qu'ils désirent croire. Aide-toi et le ciel t'aidera. » (Veyne, 1983, p. 94).

À ce premier programme pour penser la croyance, nous aimerions ajouter un principe de précaution que nous empruntons à Bruno Latour. 
Dans sa Petite réflexion sur le culte moderne des dieux faitiches, il nous invite à la vigilance :

Si nous acceptons de nous laisser instruire par ceux qui ne croient
pas en la croyance, nous nous apercevons que les modernes
n’y croient pas plus que les nègres de la côte. S'ils accusent
les sauvages de fétichisme, les blancs ne sont pas pour autant
de naïfs anti-fétichistes. Le croire serait tomber de Charybde en
Scylla. Nous aurions sauvé les nègres de la croyance - devenue
maintenant une accusation portée par les blancs sur quelque
chose qu'ils ne comprenaient pas - mais nous plongerions les
blancs dans un abîme de naïveté. Ils croiraient que les autres
croient! (Latour, 1996, p. 24)

Ma réflexion part de cette dernière phrase. Ne jamais être ni un noir, ni un blanc qui croit que l'autre croit. Il s'agit là d'une position scientifique fondamentale : la fin des anthropologies coloniales, la fin du paradigme sociologique de l'aliénation des effets et des influences, la fin d'une sémiologie structurale qui a parfois oublié les conditions de production et les conditions de réception des grands récits qui nous réunissent. Ce déplacement du chercheur en sciences de l'homme et de la société nous est dicté par la nature même de nos langages et de la complexité des réceptions qu'ils nous imposent. En observant l'aliénation de l'individu face aux pouvoirs, puis en observant la structure des signes et leurs arbitraires, les sciences sociales du $\mathrm{XX}^{\mathrm{e}}$ siècle avaient oublié d'observer et de comprendre la liberté de chacun face à sa société, et face aux langages qui conditionnent les récits de sa ou de ses communautés. Dans les mouvements des cultures numériques et créatives où nos récits prennent place aujourd'hui, nous devons envisager une plasticité de la croyance qui reflète celle des objets médiatiques, industriels, culturels et artistiques complexes qui nous sont proposés. Dans ce programme pour penser la croyance, donc, ne jamais perdre de vue que personne n'est dupe si l'on y réfléchit un peu.

À ce programme d'historien et d'anthropologue, associons enfin la psychanalyse et la sémiologie pour décrire nos modes de réception des langages et plus largement des récits qu'ils nous racontent pour qu'ensemble nous soyons et pour qu'ensemble nous croyons. Le déni, c'est cette force de l'esprit qui consiste à dire : " je sais bien, mais quand même... ». Rapporté aux langages de l'art, des médias, des industries culturelles, à nos récits, à nos images, le récepteur est dans 
un état de grâce dans lequel il peut se dire : « je sais bien que c'est une traduction, je sais bien que c'est historiquement et culturellement codé, je sais bien que ces langages me racontent des histoires, mais quand même je vais adhérer à cette proposition que l'on me fait pour appartenir confortablement aux récits de ma société ». Et les langages qui se sont toujours construits dans le double registre du factuel et du fictionnel, de la realia et de la fantasia, du réel et de l'imaginaire, ont toujours été interprétés dans cette jubilation de la croyance : « je sais bien combien tout cela est provisoire, circonstancié, joué, conventionnel, intentionnel, mais quand même, quelle belle "histoire vraie" c'est là. » Le croyant, et plus largement les croyances, demandent donc une acuité de la part de son observateur qui doit sans cesse avoir un petit sourire aux lèvres. La passion de l'un, l'investissement de l'autre, la conviction de celuilà ne sont pas des idioties, même si la passion, l'investissement et la conviction sont parfaitement joués. En 1964, dans la revue Les Temps Modernes, Octave Mannoni publie le texte fondateur de ce que nous pourrions appeler l'école française du déni (en allemand Verleugnung) (Mannoni, 1964). Ce texte, publié ensuite en 1969 dans les Clefs pour l'Imaginaire ou l'Autre Scène, a pour titre : Je sais bien mais quand même (Mannoni, 1969, p. 9-33). Inspiré par la théorie freudienne du fétichisme, Octave Mannoni se place entre psychanalyse, ethnologie et anthropologie. Il écrit :

Il faut en somme que la croyance survive au démenti, bien qu'elle devienne insaisissable, et qu'on en voie que les effets tout à fait paradoxaux. Cet exemple ouvrirait sur toutes sortes de chemins : l'utilisation de fausses nouvelles dans un but de propagande, même quand elles doivent être démenties, les offres de gascon, la psychologie du canular, et celle des imposteurs. (Mannoni. 1969, p. 23).

Le dernier thème du programme « multisciences de la croyance », nous l'empruntons à Michel de Certeau. Il écrit : « À titre de première approximation, j'entends par croyance non l'objet du croire (un dogme, un programme, etc.), mais l'investissement des sujets dans une proposition, l'acte de l'énoncer en la tenant pour vraie - autrement dit, une modalité de l'affirmation et non pas son contenu » (de Certeau, 1990, p. 260). Il poursuit : «En fait (l'histoire comme la sémiologie le montrent), l'investissement du croire passe de mythe en mythe, d'idéologie en idéologie, ou d'énoncé en énoncé. Ainsi la croyance 
se retire d'un mythe et le laisse quasi intact, mais désaffecté, mué en document » (de Certeau, 1990, p. 264).

Observer la bête quand elle est encore vivante. Observer l'investissement de l'émetteur et celui du récepteur, voir le souffle qu'ils y mettent, comprendre le sang du peuple qui circule dans le mythe, dans l'idéologie, dans l'énoncé. Mais se dire qu'il y a toujours du provisoire dans le croire. Que dès demain, je peux adhérer autrement, retourner ma veste, changer de dieux ou changer de bulletin de vote, surtout si ma communauté, mon groupe, ma société y trouve un intérêt essentiel pour sa survie, ou son confort. Et là, l'ancien mythe, l'ancienne idéologie, l'ancien énoncé, sans vies, gisent à même le sol. Ils ne sont plus des objets de jubilations, de négociation, de croyances, ils sont juste privés d'acteurs et méritent toute notre attention. Ainsi la croyance est-elle cette co-construction d'investissements partagés entre celui qui dit le mythe, l'idéologie, l'énoncé et qui s'institue dépositaire du récit, et celui qui l'interprète et le prolonge ainsi.

J'entends bien ici ne pas me faire traiter de naïf, d'innocent, ou d'aveugle. Je croirais en l'humanité ? De la croyance je ne fais pas un objet pervers? J'ai lu, il est vrai, des textes sur l'art de faire croire, sur le paradigme de la manipulation, sur l'aveuglement des hommes quand leurs croyances seraient trop fortes. On m'oppose souvent les actes terroristes, la vie sacrifiée au profit d'un groupe, une immense idiotie des publics. J'avoue parfois entendre dans Masse et puissance d'Elias Canetti (1960) comme un grand poème sur la violence des totalitarismes et la compromission des foules, comme une présomption de crétinisme généralisé si possible accusant les peuples, les masses, les publics... J'ai toujours à l'esprit le travail de Victor Klemperer, LTI, La langue du IIIe Reich, carnets d'un philologue (1966), où l'on voit comment une langue peut s'inventer pour faire régner l'intolérance et la dictature'. Il s'agit là pour moi d'une autre histoire, grave, mais qui n'a rien à voir avec la croyance telle que je l'interroge ici. Il est vrai qu'au nom du Christ on a pu massacrer sans complexe, il est vrai qu'au nom du Père on a occis plus d'une tête, il est vrai qu'au nom d'Allah on peut faire la guerre. Mais je ne suis pas historien des guerres de religions si souvent faites au profit des pouvoirs politiques. Appuyons encore un peu : « Je

1 « Il va de soi qu'à son acmé, la LTI doit être une langue de croyance, puisqu'elle vise au fanatisme » (Klemperer, 1996, p. 152). 
ne crois pas qu'une conviction religieuse ait jamais été à l'origine d'un conflit armé et sanglant, mais pense plutôt que des intentions guerrières et meurtrières ne se sont jamais servies des opinions religieuses que comme des prétextes à des fins politiques ou d'extension du pouvoir » (Rosset, 2001, p. 88). Mon travail de sémiologue consiste modestement à observer comment nous nous racontons nos histoires au quotidien, et combien la croyance est une jouissance. Comment nous nous racontons nos histoires dans le double du langage. Ce double heureux où une part du lecteur sait (qu'il s'agit d'une traduction, qu'il œuvre dans le sens de l'interprétation, que le contexte historique et culturel favorise son adhésion, que les formes et la rhétorique des textes et des images sont sans cesse réinventées pour mieux briller à chaque apparition, que les institutions sont parties prenantes dans nos mouvements et dans nos gestes...) mais ne veut rien savoir de tout cela chaque jour, chaque heure, chaque minute, sans quoi il serait transformé en 1'" homniscient », personnage sinistre, scientifique lui-même mué en document ! Nous ne travaillons donc pas sur l'art de faire croire (pas ici, pas maintenant) mais sur l'art d'être croyant.

\section{Arrête ton cinéma}

«Ce qu'il faut pour la plénitude esthétique de l'entreprise écrit André Bazin à propos du cinéma, c'est que nous puissions croire à la réalité des événements en les sachant truqués » (Bazin, 1975, p. 56). Pascal Bonitzer ajoutera plus tard : « Le cinéma présente ainsi, au niveau de la réalité, une sorte de schize qu'il faudrait conjurer d'un désaveu : c'est la racine du fétichisme » (Bonitzer, 1982, p.10). Christian Metz, dans Le signifiant imaginaire, a porté sur cet « exercice du rite cinématographique » un regard amusé. "La fréquentation des salles suppose chez le spectateur la capacité régulière et renouvelable à adopter devant ce qu'il voit une attitude où la croyance et l'incroyance se dosent avec subtilité, selon un régime de perception clivé qui n'est pas sans rapport avec le désaveu fétichiste mis au jour par Freud », écrivait-il.

Le mode de croyance cinématographique repose sur un mouvement d'aller-retour dont l'étrangeté nous est masquée par l'habitude : le film convoque la réalité, le « monde » ou son crédible substitut, mais c'est au profit de l'imaginaire ; il tisse de ressemblantes effigies le fil même de la fable et réveille 
ainsi nos désirs anciens, réveille le petit personnage épris de livres d'images et qui demandait, chaque soir, qu'on vienne lui raconter une belle histoire (Metz, 1993/1977, p. 10).

Plonger et refaire surface. L'état de vacance du spectateur ou du lecteur pourrait être rapporté à l'enfance. Cette " plénitude esthétique de l'entreprise » dont parle André Bazin, c'est donc croire et savoir en même temps. Avoir prise sur le croire et avoir prise sur le savoir, c'est un jeu d'enfant. Aller au cinéma commence par cette petite réjouissance où l'on se sait aller au cinéma : déjà nous acceptons de nous mettre en état de croyance même si nous savons que notre adhésion ne sera que provisoire pour une part. Parmi les plaisirs du cinéma, il y a celui d'aller à l'école de la croyance. On a beau dire : «Non » (non, je n'y crois pas ; non, non, ce n'est pas vrai ; non, non, non, je sais comment tout cela est fait) c'est un « oui » qui survit.

On peut entendre chez Jean-Louis Comolli cet héritage de la question du spectateur du cinématographe laissée par André Bazin, Christian Metz et Pascal Bonitzer : " Croire, ne pas croire, ne plus croire, croire malgré tout ce qui dément la croyance - ce sont les questions du cinéma » (Comolli, 2004, p. 9). Dans la vie, quand l'un d'entre nous en fait un peu trop, quand son personnage submerge sa personnalité, on lui dit sans méchanceté « Hé, arrête ton cinéma »! Ce petit avertissement qui vient de l'école du cinéma s'invite dans le réel comme un écran d'avertissement, comme un générique qui nous donnerait l'un des modes d'emploi de la vie.

\section{Chacun vient au cinéma avec son cinéma}

Dans un texte intitulé Moment critique pour la critique, Serge Daney raconte :

Il y a quelques années, à Gabès, un animateur de ciné-club du sud tunisien me fit part de son désarroi. Il n'était plus rare en effet qu'après la séance, un étudiant barbu se lève et explique gravement que si l'héroïne mourait à la fin du film, ce n'était pas le fait de scénaristes mais de Dieu, qui l'avait punie pour ses péchés. Comment dans ces conditions animer un débat de ciné-club puisque de débat il n’y avait plus ? (Daney, 1997, p. 126). 
Cette intrusion d'un croire vers un autre justifierait à elle seule les précautions qu'il faut prendre pour entendre se superposer des mondes différents. La dernière phrase de Serge Daney me laisse songeur. En effet, précisément, un débat s'ouvrait. Celui de la place prise par chacun devant le récit et les langages. Le barbu de Gabès se déplace avec sa croyance et débarque en plein film avec sa valise remplie de ses récits coraniques à lui, à la grâce de Dieu. Que l'héroïne vienne à mourir et la voilà aussitôt confiée au Très Grand par son spectateur attentif. Mais le barbu n'est pas venu en âne. Il sait très bien que l'héroïne est une fiction, que le récit respecte une forme narrative, et il s'invite à cette fête du débat de ciné-club avec ses convictions. Convictions qu'il faut rendre encore plus fortes pour lui dès lors qu'elles sont contestées d'emblée par la forme même du débat du ciné-club, et par l'acte de création du réalisateur. Celui qui aurait pleuré à la mort de l'héroïne pendant le film serait-il plus apte à débattre ? Ses larmes produites par le pacte fictionnel de l'orthodoxie du spectateur du cinématographe occidental seraient-elles le seul passeport ouvrant droit au respect de sa parole ? Chacun vient au cinéma avec son cinéma.

\section{La grande illusion et son renouvellement dans les médiacultures}

Aller au cinéma, aller au théâtre, regarder la télévision, assister à un spectacle de magie, lire son journal ou un roman, autant d'activités qui nécessitent des manières de croire. Le lecteur, le spectateur, ou le consommateur négocieront avec plus ou moins de bonheur ces différentes postures qui nous invitent à des formes de croyance, selon leur histoire d'enfance à eux et comment ils se la racontent selon sa société et comment elle les forge. Mais partout nous serons ce spectateur « qui se pose en parfait incrédule devant les tours des illusionnistes, mais qui exige que l'illusion soit parfaite, sans qu'on puisse savoir qui doit être trompé » (Mannoni, 1969, p. 9 ; Marion, 1997, p. 65).

Dans son livre sur le documentaire et ses faux semblants, François Niney nous invite à faire le distinguo entre le fictionnel et le faux : « Une fiction n'est évidemment pas un faux ni un mensonge. (...) La fiction ne demande pas à ce qu'on y croie comme à la réalité ; elle est le monde du comme si » (Niney, 2009, p. 76). En effet, Avatar n'est pas un mensonge, ni La grande illusion. La fiction est avant tout une grande illusion, mais comme toute illusion elle finit par se dissiper, le 
réel s'en charge. À la définition du mot illusion dans le dictionnaire Le Robert : " Croyance erronée, qui abuse l'esprit par son caractère séduisant ", préférons l'illusion en effet comme un compromis provisoire, une croyance non pas « erronée » mais une croyance avouée. $\grave{A}$ cette première observation, François Niney ajoute que certains films se construisent tout de même comme des mensonges : « Ne sera qualifié de faux qu'un film de fiction feignant qu'il n'en est pas une » (Niney, 2009, p. 76). Elle sonne bizarrement cette dernière phrase de François Niney : le faux, c'est un (film) feignant qu'il n'en est pas une (fiction). Le faux, c'est quand le travail du déni ne peut s'accomplir. Le faux, le mensonge, c'est quand le cinéma documentaire ou le cinéma de fiction, ou toute autre production d'image, ne fait que le mâle, sorte de baroudeur du réel et de la vérité, avec son puissant objectif d'objectivité. Au contraire, le cinéma documentaire ou le cinéma de fiction ou toute production d'image qui joue et laisse jouer le spectateur dans les frontières sans cesse renouvelées de la traduction du réel refuse une lecture univoque de l'œuvre. En matière de croyance, il est difficile de séparer des genres cloisonnés, car justement la croyance demande le décloisonnement permanent des frontières entre écriture fictionnelle et écriture documentée. J'admire en ce sens les théoriciens qui passent des heures à produire des typologies dont les frontières sont si poreuses qu'elles s'écroulent aussitôt la page tournée. La vivacité du déni du langage dépend de la créativité des jeux fictifs et réalistes dont l'œuvre fait preuve. Sans cesse renouvelées, ces formes sont aujourd'hui prises dans les registres indifférenciés des médias, des industries culturelles et de l'art. Ce sont dans ces médiacultures que le cinéma d'aujourd'hui nous fait ses cinémas.

Quand je vois Batman voler au cinéma, je ne me dis pas « c'est pas vrai! », je ne me lève pas de mon fauteuil, furieux qu'on ait osé me tromper ainsi, je vole avec ${ }^{1}$. Quand je regarde les deux dernières séquences de Paris Texas de Wim Wenders, c'est inévitable, je pleure. Je n'ai jamais pensé écrire à Wim Wenders pour le traiter de menteur, j'aurais plutôt tendance à le remercier pour l'ensemble de son œuvre. Au cinéma de fiction, nous savons que nous sommes là pour éprouver la liberté des langages qui se défont de la contingence des réels. Faire de ce lieu imaginaire, pour un moment, un moment seulement, un moment

1 Batman and the Dark Knight de Christopher Nolan, 2009. 
éclairant sur le monde et sur soi. Quand je vois le film $\hat{E}$ tre et avoir ${ }^{1}$ au cinéma, je suis face à un documentaire, et je ris des bons mots de l'enfant Jojo. J'ai bien envie de prendre ce monde tel quel sans interroger ni le montage, ni le choix du cadrage, ni les figures romancées de cette classe filmée au bout des routes de l'Auvergne. J'aimerais que ces images soient vraies mais je sais très bien leur part de fiction qui leur permet d'être justes.

\section{Conclusion, possiblement}

La croyance dans la perspective des médiacultures, tient plus de l'art de vivre que de l'art d'être aveugle. Ce postulat que je sais contestable me permet tout de même de ne pas sombrer dans le paradigme des effets : nous serions tous croyants malgré nous, parce que manipulés par le langage des médias, eux-mêmes manipulés par les discours politiques, eux-mêmes dictés par les intérêts économiques. La croyance dans les perspectives d'une sémiotique des textes des médiacultures serait cette intelligence qui dès l'enfance ne cessera d'être mise à l'épreuve. Chacun a appris à négocier avec les démentis du Père Noël et les mensonges partagés dans la complicité entre des adultes et des enfants. Chacun sait qu'il nous faut en groupe accepter des histoires pas toujours exemptes d'une part de fiction. Nul n'ignore les rituels qui permettent aux groupes de se rassembler pour donner un peu plus de crédit aux récits, aux idéologies, aux mythes, aux énoncés. Et les langages que nous échangeons en société, et les crédits que nous leur accordons, sont au cœur de cette entreprise du déni du langage considéré comme acte salutaire et créatif.

On préfèrerait la rhétorique implacable de La Société du spectacle. Nous tous vendus aux marchands et à leurs langages, chacun fait de gestes publicitaires, chaque sujet soumis aux puissances du capital, et les banques en temps de crise pouvant puiser sans complexe, invitées par les États, dans les réserves des contribuables. On souhaiterait mille coups de buttoir comme dix fois la puissance de la bombe de mai 1968 ainsi que l'espérait Guy Debord pour chasser nos soumissions aux règles et aux langages des pouvoirs par l'insoumission déclarée comme art de vivre. La société dans laquelle nous vivons est toute autre. Elle s'est faite dans le quotidien des informations et des communications

1 Etre et avoir, film documentaire français réalisé par Nicolas Philibert, France, 2002. 
des démocraties libérales, elle s'est construite dans les médiacultures, c'est-à-dire dans un mélange et un exercice sans cesse éprouvé entre les différentes sphères des médias, des industries culturelles et des arts. Elle s'est exercée sur les terrains vidéo-ludiques si proches des langages de la guerre. Elle s'est faite dans le grand partage des usages que nous autorisent les arborescences virtuelles et les réseaux sociaux. Mais cet exercice quotidien fait de passages entre des faits et des fictions, entre des formes hybrides dont il nous faut sans cesse dénouer les provenances, a fini par produire l'intelligence de la réception. À nous de ne pas en faire une arme cynique : plus rien ne serait vrai, plus rien ne serait faux, nous pourrions ne plus croire en rien. Il nous faut au contraire reconnaître chaque jour, dans nos langages, les signes qui en eux défendraient encore les récits d'une humanité faite dans la reconnaissance et la tolérance de l'autre.

La croyance n'est pas une arme offerte aux négationnistes de tout bord. C'est pouvoir être spectateur, non pas indifféremment mais possiblement, de Shoah de Claude Lanzmann, de La vie est belle de Roberto Benigni, de Nuit et brouillard d'Alain Resnais ou de l'installation d'Esther Shalev Gerz sur les objets retrouvés dans les camps de concentration et d'extermination. Là, à chaque fois, la nécessité de faire le point, d'accorder notre investissement en relation avec les langages qui nous sont proposés. La société qui n'est plus celle du spectacle est celle des traductions variées, plurielles que nous apprenons à entendre comme autant de subjectivités, et nous permettant paradoxalement de défaire chaque jour, les mots et les images qui voudraient se donner pour vrais.

La question de la croyance est fondamentalement politique et morale : qui la considère comme un instrument d'oppression et de soumission sans en considérer les dimensions co-constructives se déclare sous les effets permanents et pernicieux d'une drogue quotidienne insufflée par les pouvoirs du langage. Qui considère la croyance comme une liberté de savoir et de ne pas savoir, de choisir entre une part de réel et une part de fable, de comprendre que certains de nos mythes, de nos idéologies, de nos récits et de nos énoncés sont tissés dans le double du langage (il me dit vrai, mais il est fait de faux ; il est tout faux, mais se veut vrai ; il est faits et fictions ; il est information et communication ; il est anonyme et autoritaire mais je peux remonter à ses sources et le défaire de son enveloppe ontologique) peut alors 
entendre le monde des langages comme une construction dans laquelle il a sa part de responsabilité interprétative.

\section{Références}

Adorno, T. W. (2000). Des étoiles à terre. La rubrique astrologique du Los Angeles Times. Étude sur une superstition secondaire. Paris : Exils éditeur.

Althusser, L. (1986). Idéologie et appareils idéologiques d'État (Notes pour une recherche). Sur la reproduction des conditions de la production. Paris : Éditions sociales.

Augé, M. (1997). La guerre des rêves, exercices d'ethno-fiction. Paris : Seuil.

Anonyme 1. (20 février 2010). Légende de photographie. Le Monde Magazine, 6-7.

Anonyme 2. (11 février 2010). New Delhi correspondance. Le Monde, 6.

Barthes, R. (1979). Roland Barthes par Roland Barthes, coll. Points. Paris : Seuil.

Bastide, R. (2000). Le candomblé de Bahia. Transe et possession du rite candomblé (Brésil), coll. Terre humaine. Paris : Plon.

Bazin, A. (1975). Qu'est-ce que le cinéma ? Paris : les éditions du Cerf.

Belting, H. (2004). Pour une anthropologie des images. Paris : Gallimard.

Benjamin, W. (1971). L'homme le langage la culture. Paris : Denoël Gonthier.

Bonitzer, P. (1982). Le champ aveugle. Essais sur le cinéma. Paris : Gallimard.

Broch, H. (2000). Les prisons de l'esprit. Agone, 23 (Qu'est-ce-que croire ?), 109-129.

Canetti, E. (1986). Masse et puissance. Paris : Gallimard.

Certeau, M. de. (1990). L'invention du quotidien. Arts de faire. Paris : Gallimard.

Certeau, M. de. (1987). Faiblesse de croire. Paris : Seuil.

Christin, A-M. (1995). L'image écrite ou la déraison graphique. Paris : Flammarion.

Comolli, J.-L. (2004). Voir et pouvoir. Lagrasse : Éditions Verdier.

Cieply, M. (8 novembre 2009), A Movies's Budget Pops From the Screen, The New York Times. Disponible à : http://www.nytimes.com/2009/11/09/business/media/09avatar. $\mathrm{html}$ ? pagewanted $=$ all\&_r $=0$.

Daney, S. (1997). Devant la recrudescence des vols de sacs à main, cinéma, télévision, information. Lyon : Aléas éditeur.

Dayan, D. (octobre 1992). Les mystères de la réception. Le débat, 71, 46-162.

Debord, G. (1971). La société du spectacle. Paris : Éditions Champ libre.

Didi-Huberman, G. (1992). Ce que nous voyons, ce qui nous regarde. Paris : Les éditions de Minuit.

Didi-Huberman, G. (2009). Quand les images prennent position. L'œil de l'histoire, 1. Paris : Les éditions de Minuit.

Freedberg, D. (1998). Le pouvoir des images. Paris : Gérard Monfort éditeur.

Girard, R. (1972). La violence et le sacré, coll. Pluriel. Paris : Grasset.

Glassner, J.-J. (1993). Chroniques mésopotamiennes. Paris : Belles Lettres.

Gruzinski, S. (1990). La guerre des images. Paris : Fayard.

Klemperer, V. (1996). LTI. La langue du IIIe Reich, carnets d'un philologue. Paris : Albin Michel. 
Laïdi, Z. (2000). Le sacre du présent, coll. Champs, Paris : Flammarion.

Lapouge, G. (1999). Besoins de mirage. Paris : Seuil.

Latour, B. (1996). Petite réflexion sur le culte moderne des dieux faitiches, coll. Les empêcheurs de tourner en rond. Paris : Syntelabo éditeur.

Legendre, P. (1994). Leçons III, dieu au miroir. Étude sur l'institution des images. Paris : Fayard.

Leiris, M. (2003). Miroir de l'Afrique. La croyance aux génies Zar en Éthiopie du Nord. Paris : Gallimard.

Leiris, M., (2003). Miroir de l'Afrique. La possession et ses aspects théâtraux chez les éthiopiens de Gondar. Paris : Gallimard.

Leroi Gourhan, A. (1964). Le Geste et la parole, Technique et langage. Paris : Albin Michel.

Levi-Strauss, Cl. (1962). Le totémisme aujourd'hui. Paris : PUF.

Levi-Strauss, Cl. (1996 [1952]). Le Père Noël supplicié. Pin-Balma : Éditions Sables.

Maigret, É., \& Macé, É. (2006). Penser les médiascultures. Paris : Armand Colin.

Mannoni, O. (1969 [1964]). Clefs pour 1'Imaginaire ou l'Autre Scène. Paris : Seuil.

Marion, J.-L. (1977). L'icône et la distance, coll. Biblio Essai. Paris : Le livre de poche.

Marion, Ph. (1997). Narratologie médiatique et médiagénie des récits. Recherches en communication, 7, 61-87.

Metz, Ch. (1993). Le signifiant imaginaire. Paris : Christian Bourgois éditeur. (Publication originale : 1977)

Mondzain, M.-J. (2003). L'image peut-elle tuer ? Paris : Bayard.

Niney, Fr. (2009). Le documentaire et ses faux-semblants. Paris : Klincksieck.

Peirce, Ch. S. (1978). Écrits sur le signe. Paris : Seuil.

Racamier, P. Cl. (1992). Le génie des origines. Aphorismes autour du déni, Paris : Payot.

Ricoeur, P. (2000). La mémoire, l'histoire, l'oubli. Paris : Seuil.

Rosset, Cl. (1977). Le réel, traité de l'idiotie. Paris : Minuit.

Rosset, Cl. (novembre 2001). La passion de l'idiotie. Art Press, 273, 56-58.

Schaeffer, J.-M. (1999). Pourquoi la fictions?. Paris : Seuil.

Schmitt, J.-Cl. (2001). Les corps, les rites les rêves le temps, Essai d'anthropologie médiévale. Paris : Gallimard.

Sontag, S. (1995). L'esprit comme passion. Dans C. Geoffroy (Éd.), Catalogue Elias Canetti, Paris : Bibliothèque publique d'information, Centre Georges Pompidou.

Sperber, D. (1982). Le savoir des anthropologues, coll. Savoir. Paris : Hermann.

Veyne, P. (1983). Les grecs ont-ils cru à leurs mythes. Paris : Seuil.

Warburg, A. (mars 2003). Le rituel du serpent. Art et anthropologie. Paris : Macula. 